\title{
Traumatic Ceramic Femoral Head Fracture: An Initial Misdiagnosis
}

\author{
Mohammad H. Fard-Aghaie, Mustafa Citak, Joao Correia, Carl Haasper, Thorsten Gehrke and \\ Daniel Kendoff
}

Orthopaedic Department, Helios ENDO-Klinik Hamburg, Germany

\begin{abstract}
Background and Purpose: Ceramic heads are widely used in modern total hip arthroplasty (THA). Although a rare complication, fractures of ceramic heads are described in the literature, evoking uncertainties regarding the in vivo stability of this material, especially when impaction of a fractured ceramic head can lead to disastrous results.

Methods: In this case report, we present a fracture of a ceramic head after trauma. A misinterpretation of the initial radiographs led to severe fragmentation of the ceramic head two weeks after the incident, later resulting in complete destruction of the arthroplasty.

Results and Interpretation: Remarkably, radiographs obtained more than one year after the trauma led to this delayed diagnosis. A single radiographic evaluation, especially in patients with persistent pain after major trauma, with a ceramic head in a THA, seems to be insufficient.
\end{abstract}

Keywords: Total hip arthroplasty, ceramic head fracture, misdiagnosis, complication, trauma, radiographic evaluation.

\section{INTRODUCTION}

In the 1970's, Boutin implemented ceramic in modern total hip arthroplasty (THA). Although initial fracture rates of $13.4 \%$ for ceramic heads were described before the 1990 's, the inferior rate of wear and friction when compared to metallic heads, and optimized tribology was very promising in THA [1-3]. Gradual improvements in processing of the material led to a significant reduction of the fracture rate to below $0.1 \%$ [3]. Thus, alumina ceramic heads have currently become the standard material in THA with ceramic bearing surfaces.

Nevertheless, multiple case reports have been published describing ceramic head fractures [4-11]. The causes of fractures are diverse and vary from traumatic events $[5,9$, $12,13]$ to impingement between the neck and the liner rim [7]. Spontaneous fractures without any history of trauma have also been described $[4,6,8,10,11]$. However, only two reports describing delayed fractures of ceramic heads were found $[12,13]$.

In this report, we present a 24-year old patient who underwent primary THA at our institution and was a victim of high-energy trauma seven years later. Initial radiographs were misinterpreted in a non-designated total joint clinic at the time of primary admission (after the accident). A destructive ceramic head fracture was diagnosed more than one year after initial trauma at our institution, with major destruction of the ceramic head and the remaining THA. This was followed by an extensive revision. Based on this experience, the general question of adequate radiographic diagnosis after trauma to a THA, especially one with partial

*Address correspondence to this author at the Orthopaedic Department, Helios ENDO-Klinik Hamburg, Holstenstrasse 2, D-22767 Hamburg, Germany; Tel: 0049403197 1669; Fax: 0049403197 1200;

E-mail: Daniel.Kendoff@endo.de or full ceramic bearing surfaces, will be further discussed in this report.

\section{PATIENT AND CASE DESCRIPTION}

\section{Case}

A 24-year old patient with secondary osteoarthritis of the hips due to Legg-Calve-Perthes disease underwent primary hybrid THA of the left hip seven years before trauma, followed by primary cementless THA of the right hip one year later at our institution (Fig. 1) Six years after the right THA, he was involved in a motorcycle accident, in which he suffered direct trauma to this hip, as well as a complex ankle

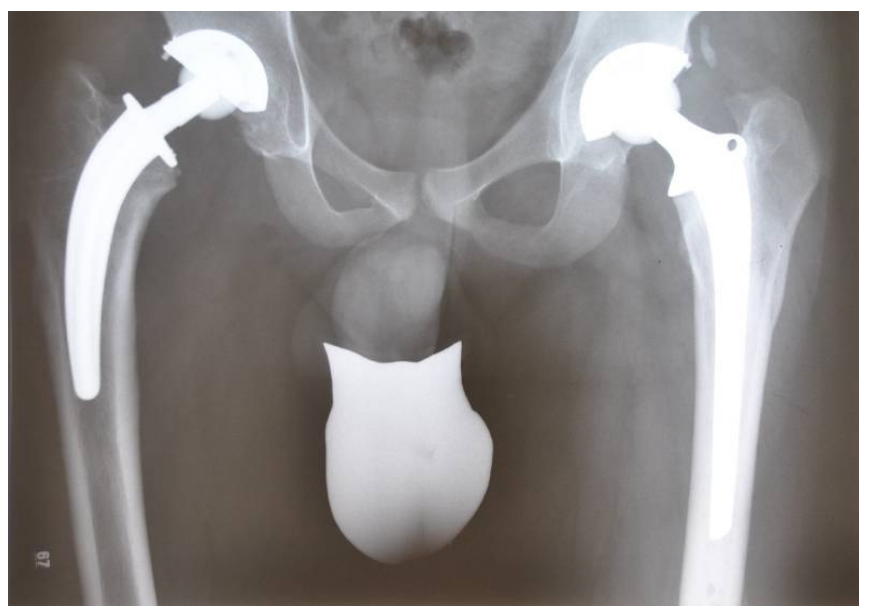

Fig. (1). Anteroposterior pelvic radiograph (24 year old male) after primary THA for secondary osteoarthritis due to Perthes disease (Right Hip: cementless CFP stem and TOP acetabular cup; Left Hip: Hybrid THA with cemented ENDO Mark III stem + cementless TOP acetabular cup, Waldemar Link GmbH, Hamburg, Germany). 
fracture which was treated in a smaller regional hospital on admission. Although initial anteroposterior radiographs of the pelvis and right hip axial views were performed, no signs of fractures, aseptic loosening or implant damage were diagnosed by the attending trauma surgeons (Fig. 2). Two weeks after surgical treatment of the ankle fracture, the patient noticed a sudden "cracking" sound in his right hip, as well as shortening of the right lower limb. Interestingly, the patient had no relevant pain.

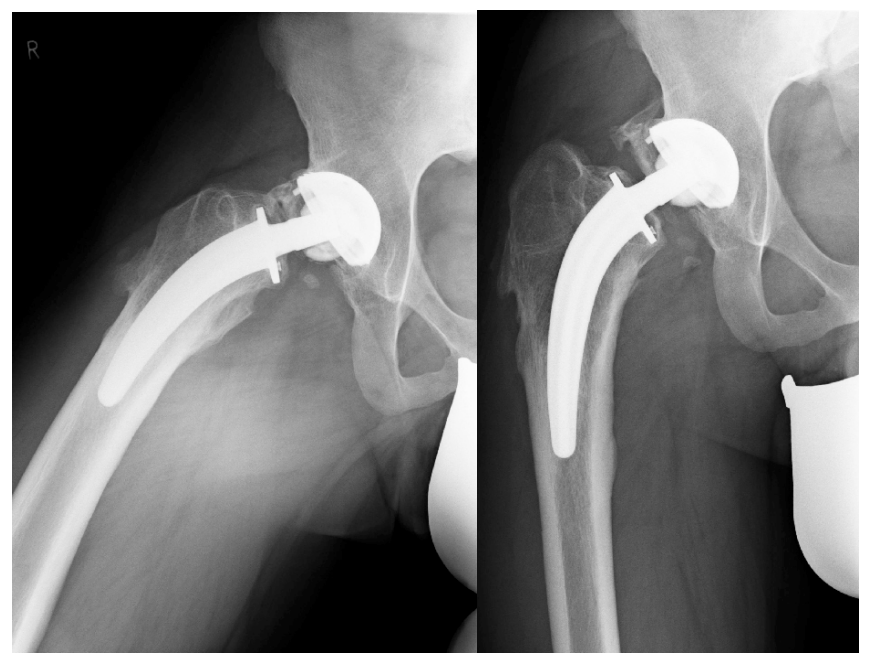

Fig. (2). Conventional pelvic radiographs directly after trauma, revealing ceramic head fracture.

Despite this sign, he had no further medical observation or secondary radiographic evaluation over the next month. In the following months he was increasingly troubled by back pain and an unpleasant cracking "phenomenon" with movement of his right hip; no severe hip pain or associated direct thigh pain was mentioned at any time. Further conventional radiographs, in two planes, more than a year after the initial radiographs, revealed a completely destroyed multifragmented ceramic head (Fig. 3). A thorough clinical examination revealed a shortening of $2 \mathrm{~cm}$ of the right lower limb, yet the patient was able to walk without relevant pain.

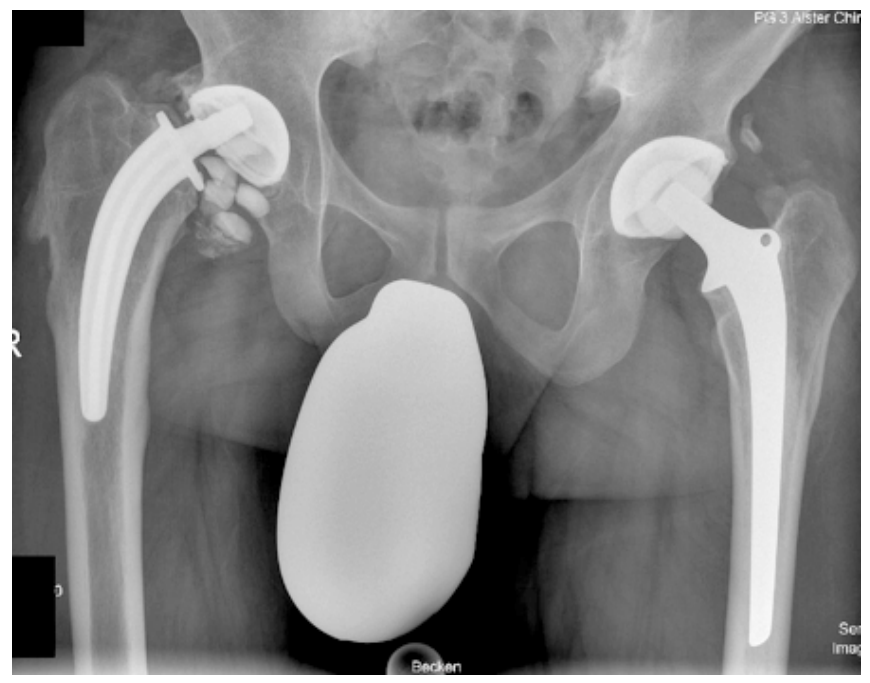

Fig. (3). Anteroposterior pelvic radiograph eight months after the initial radiographs, showing a fractured ceramic head.

\section{Surgical Revision}

Intraoperatively, extensive damage of the ceramic head and correlating taper junction of the femoral neck was found (Figs. 4, 6, 7). Concomitantly, there was severe debrisrelated metallosis throughout the entire joint (Fig. 5). The intervention consisted of conversion of the short cementless CFP stem to a cementless revision stem and an acetabular cup revision to a ceramic-ceramic bearing surface (Alloclassic stem and Allofit-IT acetabulum with Delta Ceramic Inlay, Zimmer, Warsaw, Indiana, USA and Ceramtec, Plochingen, Germany). Meticulous debridement of all affected soft tissues and extensive lavage was additionally performed.

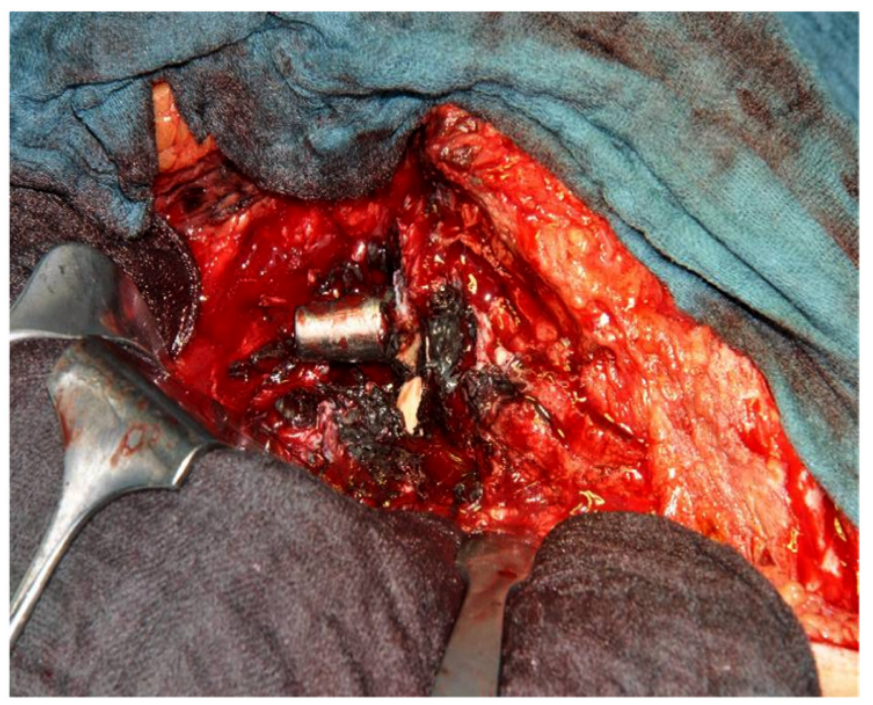

Fig. (4). Intra-operative image showing countless debris and the damaged taper junction of the femoral neck (in situ).

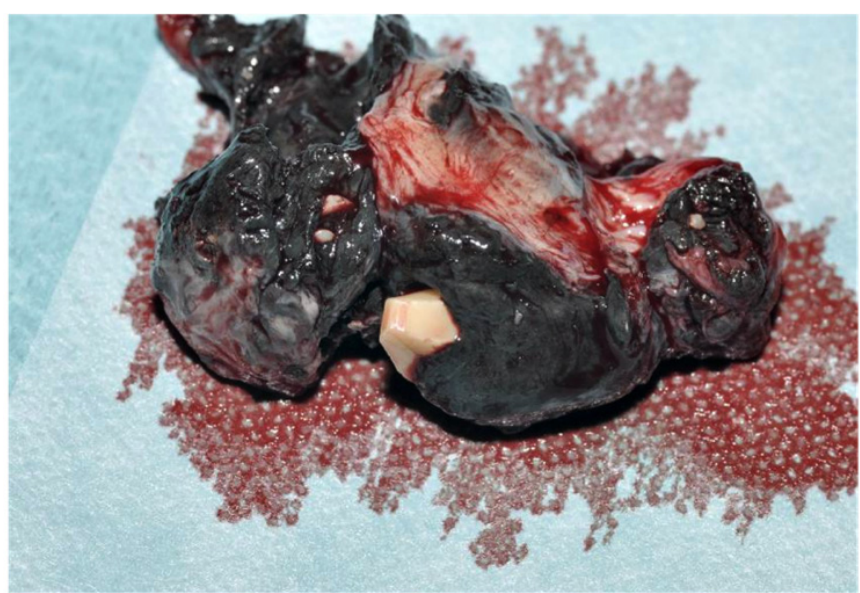

Fig. (5). Intra-operative image showing debris with ceramic fragments.

\section{Postoperative Treatment and Follow-Up}

The postoperative course was uneventful, with radiographs revealing a correct position and articulation of the cementless implant (Fig. 8). A 12 month postoperative inquiry was performed and the Oxford Hip Score obtained, with both indicating satisfactory joint function [14]. The patient had no pain and was able to perform his daily activities. 


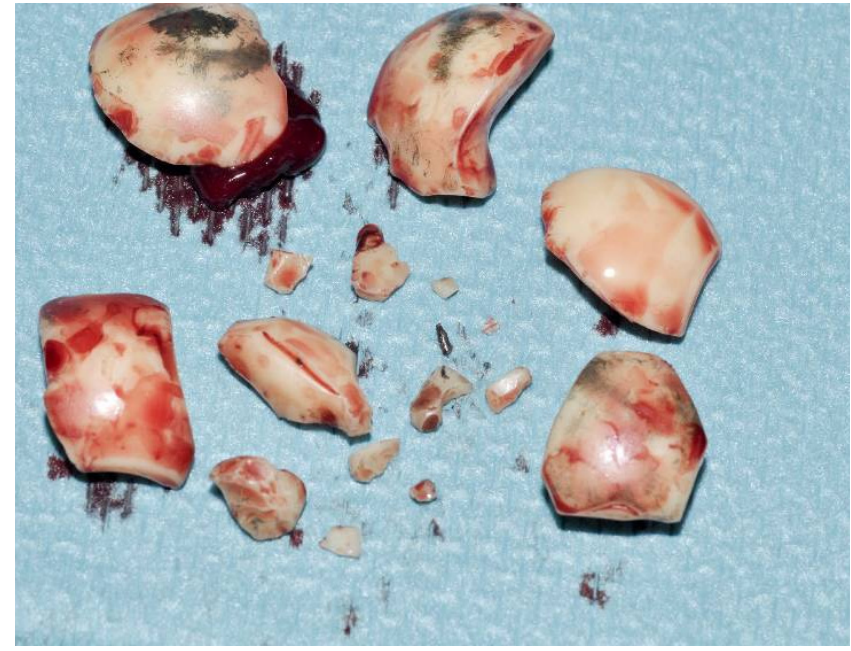

Fig. (6). Intra-operative image showing the fragmentation of the ceramic head.

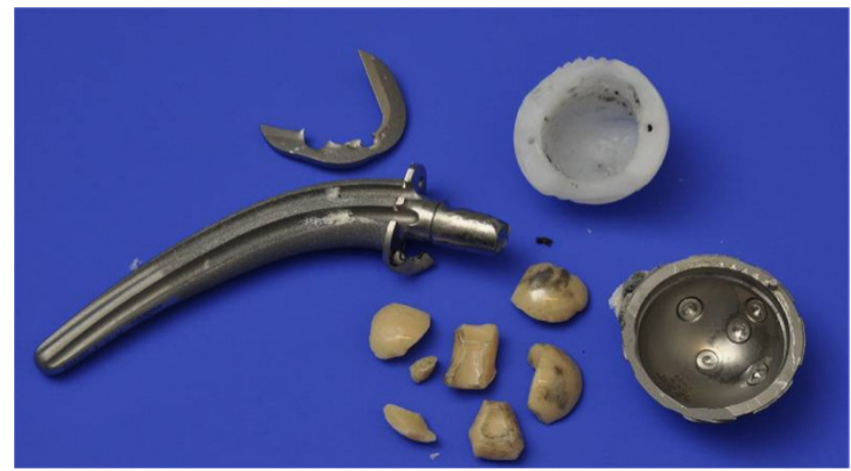

Fig. (7). Image showing the explanted components: a fragmented ceramic head, a damaged stem and acetabular cup and liner.

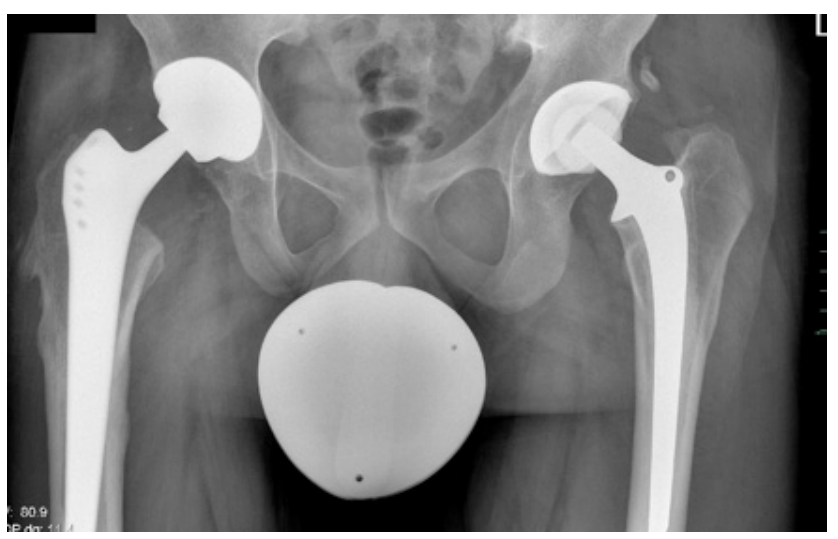

Fig. (8). Postoperative anteroposterior pelvic radiograph.

\section{DISCUSSION}

Although a relatively rare complication in modern THA, the described ceramic head fracture was misinterpreted in initial radiographs, which showed a discreet fracture of the ceramic head. Two assumptions can be made which led to this misdiagnosis. Firstly, the complex ankle fracture was probably more painful than the ceramic head fracture and this misled the surgeons. Secondly, which is more fact than assumption, is that the initial center of admission was incapable of reaching the correct diagnosis when one considers that the initial radiographs revealed a ceramic head fracture.

The "cracking sound" incident two weeks after trauma, as described by the patient, was probably due to the complete fracture of the ceramic head. Ultimately, a multifragmented ceramic head fracture was diagnosed one year later. This raises the question of the need of a possible guideline or recommendation for patients suffering acute trauma of the lower limb with a total hip arthroplasty, especially those with ceramic bearing surfaces.

This case remarkably demonstrates the variability and intensity of symptoms: when one considers the implants' damage, the patient was able to walk without any pain originating from the hip. His only complaint was mild back pain over the subsequent months. This clearly presents a challenge for the assisting physician to obtain a correct diagnosis. The posttraumatic patient who previously underwent THA should therefore be carefully followed. We suggest a close follow-up including repeat conventional radiographs several weeks after trauma. Furthermore, in some cases, a CT-Scan could provide the correct diagnosis [15]. Ultimately, if a definite diagnosis in the initial assessment center (e.g. low volume or non-dedicated joint replacement center) cannot be assured, presentation of the case to a dedicated joint replacement center should be considered.

We present our case report to increase awareness among physicians and training staff who treat trauma patients who underwent previous total joint replacements, in order to expedite the diagnosis of possible posttraumatic implant fractures/failures.

\section{ACKNOWLEDGEMENTS}

No benefits in any form have been received or will be received from a commercial party related directly or indirectly to the subject of this article. No funds were received in support of this study.

\section{CONFLICT OF INTEREST}

The author(s) confirm that this article content has no conflicts of interest.

\section{REFERENCES}

[1] Boutin P. Total arthroplasty of the hip by fritted aluminum prosthesis. Experimental study and 1st clinical applications. Rev Chir Orthop Reparatrice Appar Mot 1972; 58(3): 229-46.

[2] Fritsch EW, Gleitz M. Ceramic femoral head fractures in total hip arthroplasty. Clin Orthop Relat Res 1996; 328: 129-36.

[3] Willmann G. Ceramic femoral head retrieval data. Clin Orthop Relat Res 2000; 379: 22-8.

[4] Arenas A, Tejero A, Garbayo A, Arias C, Barbadillo J, Lecumberri J. Ceramic femoral head fractures in total hip replacement. Int Orthop 1999; 23(6): 351-2.

[5] Habermann B, Ewald W, Rauschmann M, Zichner L, Kurth AA. Fracture of ceramic heads in total hip replacement. Arch Orthop Trauma Surg 2006; 126(7): 464-70.

[6] Holmer P, Nielsen PT. Fracture of ceramic femoral heads in total hip arthroplasty. J Arthroplasty 1993; 8(6): 567-71.

[7] Hwang DS, Kim YM, Lee CH. Alumina femoral head fracture in uncemented total hip arthroplasty with a ceramic sandwich cup. J Arthroplasty 2007; 22(3): 468-71.

[8] Otsuka NY, Schatzker J. A case of fracture of a ceramic head in total hip arthroplasty. Arch Orthop Trauma Surg 1994; 113(2): 812 . 
[9] Rhoads DP, Baker KC, Israel R, Greene PW. Fracture of an alumina femoral head used in ceramic-on-ceramic total hip arthroplasty. J Arthroplasty 2008; 23(8): 1239 e25-30.

[10] Sharma V, Ranawat AS, Rasquinha VJ, Weiskopf J, Howard H, Ranawat CS. Revision total hip arthroplasty for ceramic head fracture: a long-term follow-up. J Arthroplasty 2010; 25(3): 342-7.

[11] Toran MM, Cuenca J, Martinez AA, Herrera A, Thomas JV. Fracture of a ceramic femoral head after ceramic-on-ceramic total hip arthroplasty. J Arthroplasty 2006; 21(7): 1072-3.
[12] Anwar I, Bhatnagar G, Atrah S. Delayed catastrophic failure of a ceramic head in hybrid total hip arthroplasty. J Arthroplasty 2009; 24(1): 158 e5-8.

[13] McLean CR, Dabis H, Mok D. Delayed fracture of the ceramic femoral head after trauma. J Arthroplasty 2002; 17(4): 503-4.

[14] Dawson J, Fitzpatrick R, Carr A, Murray D. Questionnaire on the perceptions of patients about total hip replacement. J Bone Joint Surg Br 1996; 78(2): 185-90.

[15] Toni A, Traina F, Stea S, et al. Early diagnosis of ceramic liner fracture. Guidelines based on a twelve-year clinical experience. J Bone Joint Surg Am 2006; 4: 55-63.

(C) Fard-Aghaie et al.; Licensee Bentham Open.

This is an open access article licensed under the terms of the Creative Commons Attribution Non-Commercial License (http://creativecommons.org/licenses/by-nc/3.0/) which permits unrestricted, non-commercial use, distribution and reproduction in any medium, provided the work is properly cited. 